\title{
Percutaneous epididymal sperm aspiration and short time insemination in the treatment of men with obstructive azoospermia
}

\author{
Yan Jiang • Qinying Cao $\cdot$ Xiujun Zhao $\cdot \mathrm{Li} \mathrm{Li} \cdot$ \\ Shuxian Li • Fulu Gao
}

Received: 5 May 2013 / Accepted: 5 August 2013 / Published online: 29 August 2013

(C) Springer Science+Business Media New York 2013

\begin{abstract}
Objective To study the efficacy of percutaneous epididymal sperm aspiration (PESA) in combination with short time insemination to treat infertile men with obstructive azoospermia (OA).

Design Paired randomized controlled trial in which each couple's cohort of oocytes was divided into two equal groups.

Setting Center for reproductive care.

Patients Twenty men with OA.

Interventions Motile spermatozoa were collected using PESA. Half of the oocytes were used for intracytoplasmic sperm injection (ICSI). The rest were inseminated briefly with PESA sperm in vitro fertilization (IVF). After 4-5 h, the remaining cumulus cells were removed mechanically for second polar body observation to decide whether to apply "rescue" ICSI (RE-ICSI).
\end{abstract}

Main outcome measures Rates of oocyte maturation, fertilization, cleavage, and good quality embryos. Numbers of available embryos and good quality embryos were compared between PESA-IVF (using a short incubation protocol+rescue ICSI) group and PESA-ICSI group.

Results In the short time insemination group, cumulus cells were dispersed by PESA spermatozoa. No second polar bodies were found, so RE-ICSI was done. PESA-IVF + RE-ICSI and PESA-ICSI outcomes were comparable in terms of fertilization rates, $2 \mathrm{PN}$ cleavage rate and good quality embryo rates with no statistically significant differences.

Conclusions PESA sperm without centrifugation could disperse the cumulus cells but were infertile and therefore could substitute for synthetic hyaluronidase. The outcomes of PESA-IVF with rescue ICSI were equivalent to PESA-ICSI. Using spermatozoa obtained by PESA and IVF before REICIS is a viable treatment for men with OA.

Keywords Percutaneous epididymal sperm aspiration . Obstructive azoospermia $\cdot$ Short time insemination $\cdot$ Rescue ICSI $\cdot$ Hyaluronidase $\cdot$ Male infertility

\section{Introduction}

Obstructive azoospermia (OA) is characterized by an occlusion or partial absence of the male reproductive tract with the presence of normal spermatogenesis [1]. The standard treatment for infertile men with OA, whether congenital or acquired, is percutaneous epididymal sperm aspiration (PESA) followed by intracytoplasmic sperm injection (ICSI) [2, 3] . Achieving pregnancies and the birth of normal, healthy infants using epididymal spermatozoa together with conventional in vitro fertilization (IVF) [4-6] or intrauterine insemination (IUI) [7-9] indicates that the epididymal sperm can fertilize both in vitro and in vivo. 
In 1985, Temple-Smith [4] reported the first pregnancy with the use of spermatozoa obtained by microsurgical epididymal sperm aspiration (MESA) and IVF. In 1988, proximal (caput) epididymal sperm were demonstrated to have better motility than senescent sperm in the distal (cauda) epididymis, and it was thought that retrieval of motile sperm from the caput would yield reliable fertilization and pregnancy rates after conventional IVF [5]. As an alternative to MESA, a simplified method, PESA was introduced for retrieval of epididymal spermatozoa in patients with OA $[10,11]$. However, the numbers of aspirated epididymal spermatozoa are generally low after MESA/PESA compared with ejaculated spermatozoa. The fertilization rate using MESA/PESA in combination with conventional IVF has been low [12], but since the introduction of ICSI [13], fertilization rates with the use of epididymal spermatozoa after PESA have improved [14].

We have used short time insemination for couples with primary infertility, with unexplained infertility, or where the male partner has mild oligozoospermia or asthenozoospermia to avoid low fertilization rates or complete fertilization failure $[15,16]$. The cumulus cells were partially dispersed by the spermatozoa instead of by conventional hyaluronidase treatment at $6 \mathrm{~h}$ of insemination for observing oocyte maturation and second polar body extrusion [16]. Oocytes were subjected to early rescue ICSI (RE-ICSI) if there was fertilization failure or a low fertilization rate $[16,17]$. PESA in combination with conventional IVF gives low fertilization rates. Therefore, we chose those patients with $\mathrm{OA}$ and adequate sperm motility obtained from PESA using short time insemination and RE-ICSI.

\section{Patients and methods}

\section{Patients}

From March 2012 to May 2013, infertile patients at Shijiazhuang No. 4 Hospital were evaluated. Men with an azoospermic ejaculate, with absent or low fructose associated with nonpalpable vasa deferentia were diagnosed with OA. Clinical histories, physical examinations, and measures of serum hormone levels were obtained preoperatively. Laboratory endocrinology assays for serum follicle-stimulating hormone (FSH) and testosterone levels were within the normal range. The combination of short time insemination and sperm retrieval was offered to couples with male factor infertility caused by bilateral OA. The protocol and potential risks and benefits of the study were explained to participants before they provided written informed consent. Institutional ethics committee approval was obtained, all subjects were informed of the study and written informed consent was obtained from each subject prior to the performance of any study procedures.

\section{PESA}

For PESA, a 26-gauge needle attached to a 1-mL tuberculin syringe was inserted through the scrotal skin into the epididymis. Negative pressure was created by pulling the syringe plunger while the tip of the needle was moved gently in and out of the epididymal duct until a clear fluid was seen coming into the syringe. The aspirate was flushed into a tube containing warm sperm medium (US ART products) and taken to the laboratory for immediate microscopic examination [1]. It has been recommended to commence aspiration at the caput and corpus of the epididymis because aspirates from the cauda usually contain poor quality senescent spermatozoa, debris and macrophages $[18,19]$. The epididymal aspirates were diluted in $1 \mathrm{ml}$ IVF medium at room temperature. Good quality motile spermatozoa found in PESA aspirates were included, so we did not centrifuge them. Aspirates with lots of debris, macrophages or red blood cells were excluded.

\section{ICSI and short time insemination plus RE-ICSI}

We applied this protocol whenever the required quantity of motile sperm was collected by PESA and more than 10 oocytes were collected. The sibling oocytes were randomized into two groups. Half of the oocytes used for ICSI were treated with bovine hyaluronidase (Sigma-Aldrich, St Louis, MO, USA) for dispersing the cumulus cells $5 \mathrm{~h}$ after oocyte collection followed by ICSI, which was performed according to Esteves et al. [2,3]. The remaining oocytes were mixed with PESA sperm for short time insemination $2-2.5 \mathrm{~h}$ after collecting the oocytes and the cumulus cells were removed mechanically after $4-5 \mathrm{~h}$ co-incubation for polar body observation to decide whether to perform RE-ICSI [17].

Embryos were cultured in Quinn's sequential culture medium (US ART products) with $5.5 \% \mathrm{CO}_{2}$ at $37{ }^{\circ} \mathrm{C}$. The ICSI fertilization rate was defined as the number of fertilized oocytes based on the numbers of pronuclei $(\mathrm{PN})$ and completion of meiosis II (MII) as (number of $1 \mathrm{PN}+2 \mathrm{PN}+$ more $\mathrm{PN}+$ late cleavage zygotes)/number of MII oocytes. The number of "available" embryos was defined as the number of embryos transferred on day 3 of culture plus all cryopreserved embryos. "Good quality embryos" should have 7-8 cells on day 3, contain less than $20 \%$ fragments, but might be a little uneven in appearance. The "good quality embryo rate" was defined as the number of quality embryos divided by the number of available embryos [20]. Rates of oocyte maturation, fertilization, cleavage, and good quality embryos, and numbers of available embryos and good quality embryos were compared between the groups. 
Data analysis

Analyses were performed using the SPSS 13.0 statistical software package (SPSS Inc., Chicago, IL, USA). The results are presented as the mean values $( \pm$ SD). Differences between the groups were analyzed using Student's $t$ or $\chi^{2}$ tests and $P<0.05$ was considered statistically significant.

\section{Results}

The patients' mean age was $25.6 \pm 5.3$ years, the female partners ranged in age from 22 to 32 years with a mean duration of primary infertility of $4.1 \pm 4.7$ years. Twenty couples were included with a total of 282 oocytes and 141 oocytes in each treatment group. The cumulus cells had been dispersed by the PESA spermatozoa and were easy to remove after $4 \mathrm{~h}$ in the IVF short time insemination group. No oocytes with a second polar body were found so these oocytes were subjected to RE-ICSI. Therefore, we regarded the short time insemination group as the REICSI group. Comparing the two groups, the numbers of MII oocytes, ICSI and RE-ICSI fertilization rates, cleavage rates and numbers of available embryos were similar. There were more good quality embryos and higher good quality embryo rate in the RE-ICSI group than in the ICSI group, but the results had no statistical significance (Table 1).

PESA sperm without centrifugation could disperse the cumulus cells but were infertile and therefore could be used as a substitute for synthetic hyaluronidase. The outcomes of PESA-IVF with rescue ICSI is equivalent to PESA-ICSI. Thus spermatozoa obtained by PESA plus short time insemination and RE-ICSI is a valid treatment for men with $\mathrm{OA}$.

Table 1 Comparison of two groups of twenty OA patients

\begin{tabular}{lccl}
\hline & ICSI group & RE-ICSI group & $P$ \\
\hline Oocytes & 141 & 141 & \\
MII oocyte rate (\%) & $85.8(121 / 141)$ & $87.2(123 / 141)$ & 0.728 \\
Fertilization rate (\%) & $85.1(103 / 121)$ & $87.0(107 / 123)$ & 0.674 \\
1PN rate (\%) & $2.5(3 / 121)$ & $2.4(3 / 123)$ & 0.948 \\
2PN rate (\%) & $76.0(92 / 121)$ & $78.0(96 / 123)$ & 0.709 \\
>2PN rate (\%) & $3.3(4 / 121)$ & $3.2(4 / 123)$ & 0.918 \\
Late cleavage rate (\%) & $3.3(4 / 121)$ & $3.2(4 / 123)$ & 0.918 \\
2PN cleavage rate (\%) & $97.8(90 / 92)$ & $96.9(93 / 96)$ & 0.686 \\
Available embryos & 85 & 87 & \\
Good quality embryos & 42 & 46 & \\
Good quality embryo rate (\%) & $49.4(42 / 85)$ & $52.9(46 / 87)$ & 0.651 \\
\hline
\end{tabular}

\section{Discussion}

Causes of OA are classified into two groups; acquired and congenital. Acquired OA include vasectomy, failure of vasectomy reversal, post-infectious disease, surgical procedures in the scrotal, inguinal, pelvic or abdominal regions, and trauma $[2,21]$. Congenital causes of OA include cystic fibrosis, congenital absence of the vas deferens (CAVD), ejaculatory duct or prostatic cysts, and Young's syndrome [3, 21].

Treatment of patients with OA is usually using PESA to get enough sperm to perform ICSI. Hirsh [18] reported that adequate sperm preparations were obtained in 46/50 treatment cycles $(92 \%)$ of patients with OA with the best motility found in the caput epididymis in $89 \%$ of cases. In another study, the PESA sperm count was adequate $(\geq 20 \times 106 / \mathrm{ml})$ in $13.8 \%$ of cases and $20 \%$ sperm motility was obtained in $15.5 \%$ of sperm retrievals [19]. Here, aspirates with lots of debris, macrophages or red blood cells were excluded; only good quality motile spermatozoa were included, so we did not centrifuge them.

Spermatozoa approaching the oocyte have to overcome three main obstacles: the cumulus-corona complex, the zona pellucida and the oolemma. The cumulus-corona complex is composed of granulose-derived cumulus cells embedded in a matrix of hyaluronan oligosaccharide chains cross-linked by hyaluronan binding proteins and proteoglycans, and surrounds each oocyte [22]. In IVF, acrosomal enzymes of the inseminated spermatozoa can disperse the cumulus cells and most of the spermatozoa have the ability of fertilization. Oocyte activation is judged by the presence of two polar bodies following cumulus cell removal at $4 \mathrm{~h}$ after insemination [23]. The mean epididymal sperm fertilization rate was only $11.2-19 \%$ using conventional IVF [12]. Those fertilization failures after conventional IVF appeared to be because of the inability of spermatozoa to penetrate the zona pellucida rather than to an inability to reach the zona [24]. Therefore, PESA-derived spermatozoa can disperse the cumulus cells instead of hyaluronidase, so we can use them for PESA-IVF (using a short incubation protocol+rescue ICSI). Epididymal sperm possess fertilization capacity after centrifugation not only in vivo [6-9] but also in vitro [4, 5]. However, the results of this study demonstrate that epididymal sperm have no fertilization ability without centrifugation. The reason is unknown; perhaps centrifugation may have a "washing" effect that helps to remove any coating "decapacitation factors" that activate the spermatozoa for penetrating the zona pellucida.

Because of the low fertilization rate of PESA-IVF, low pregnancy rate of PESA-IUI, and the implementation of ICSI technology, almost all OA patients were treated with ICSI directly. Recently, RE-ICSI has become a reasonable method for patients with total fertilization failure (TFF) or low fertilization rate [23]. Hence, we considered trialing a combination of PESA-IVF and RE-ICSI a viable treatment for OA. Since 
ICSI was first developed, bovine or ovine hyaluronidase has been used routinely for oocyte denudation [25]. This enzyme digests the hyaluronic acid interspaced between the cumulus cells [26], thus liberating the oocyte for maturity grading and microinjection. To reduce unknown harmful effects from the enzyme, hyaluronidase preparation, low enzyme concentrations and short time incubation of the cumulus-oocyte complexes are recommended [25]. Animal-derived products have limited purity and accidental transmission of pathogens cannot be excluded fully. These limitations inevitably imply an increased need for mechanical dispersal of the cumulus and corona by means of multiple pipetting, which could be harmful to the oocyte [27]. Evison and De Vos reported that the use of human recombinant hyaluronidase could improve ICSI survival and good embryo development compared with bovine hyaluronidase $[27,28]$.

There were two main differences between the ICSI and REICSI groups: (1) the cumulus cells were dispersed by PESAderived spermatozoa in RE-ICSI group, reducing the potential harmful effects of hyaluronidase in the ICSI group; and (2) the timing of microinjection. Microinjection time for the ICSI group was about $1 \mathrm{~h}$ earlier than that of the RE-ICSI group. Traditional RE-ICSI is done slightly later $(6 \mathrm{~h}$ after short insemination) to prevent a delay in second polar body extrusion. Here, the $>2 \mathrm{PN}$ fertilization rate was similar in the two groups and indicates that PESA sperm without centrifugation have no fertilization ability. Therefore, it was feasible to conduct RE-ICSI a little earlier ( $4 \mathrm{~h}$ after short insemination). RE-ICSI performed earlier could result in better outcomes in the select group of patients [29] and it has been shown that the good quality embryo rate of a RE-ICSI group can be higher than that in an ICSI group when using ejaculated spermatozoa [30]. Previous studies showed that epididymal sperm from patients with $\mathrm{OA}$ and with a longer epididymis have a higher IVF rate than from those with a shorter epididymis [31, 32]. However, there was no difference between these types of men when doing ICSI [33].

Unique features of our study include using motility spermatozoa acquired from PESA without centrifugation, a short period insemination to disperse the cumulus cells and simulate physiological conditions, and RE-ICSI performed as early as possible when no second polar body was found. The limitations of this study are that the number of cases was too few to reach any significant conclusions and the data require validation with later clinical outcomes (e.g. clinical pregnancy rates).

Compared with PESA-ICSI, the procedure of PESA-IVF plus RE-ICSI can disperse the cumulus cells but needs enough motile sperm. The outcomes of sibling oocytes separated into two groups are equivalent. The PESA-IVF plus RE-ICSI procedure is more "complicated" than using ICSI directly but can offer a feasible method for the treatment of men with OA.

\section{References}

1. Esteves SC, Miyaoka R, Agarwal A. Sperm retrieval techniques for assisted reproduction. Int Braz J Urol. 2011;37(5):570-83.

2. Esteves SC, Agarwal A. Sperm retrieval techniques. In: Gardner DK, Rizk BRMB, Falcone T, editors. Human assisted reproductive technology: future trends in laboratory and clinical practice. Cambridge: Cambridge University Press; 2011. p. 41-53.

3. Male Infertility Best Practice Policy Committee of the American Urological Association, Practice Committee of the American Society for Reproductive Medicine. Report on evaluation of the azoospermic male. Fertil Steril. 2006;86 Suppl 1:S210-5.

4. Temple-Smith, Southwick, Yates, et al. Human pregnancy by in vitro fertilization (IVF) using sperm aspirated from the epididymis. In Vitro Embryo Trans. 1985;2:119-22.

5. Silber SJ, Balmaceda J, Borrero C, Ord T, Asch R. Pregnancy with sperm aspiration from the proximal head of the epididymis: A new treatmeat for congenital absence of the vas deferens. Fertil Steril. 1988:50:525-8.

6. Patrizio P, Silber S, Ord T, Balmaceda JP, Asch RH. Two births after microsurgical sperm aspiration in congenital absence of vas deferens. Lancet. 1988;10(8624):1364.

7. Qiu Y, Yang DT, Wang SM, Sun HQ, Jia YF. Successful pregnancy and birth after intrauterine insemination using caput epididymal sperm by percutaneous aspiration. Asian J Androl. 2003;5(1):73-5.

8. Qiu Y, Wang SM, Yang DT. Percutaneous vasal sperm aspiration and intrauterine insemination in the treatment of obstructive azoospermia. Fertil Steril. 1997;68(6):1135-8.

9. Chatterjee S, Chaudhuri AR, Chowdhuri RG, Rahman S. Percutaneous epididymal sperm aspiration followed by intrauterine insemination-a new approach to achieve a pregnancy in infertility due to obstructive azoospermia. J Indian Med Assoc. 2001;99(8):445-7.

10. Craft I, Shrivastav P. Treatment of male infertility. Lancet. 1994;344:191-2.

11. Shrivastav P, Nadkami P, Wensvoort S, Craft I. Percutaneous epididymal sperm aspiration for obstructive azoospermia. Hum Reprod. 1994;9(11):2058-61.

12. Silber SJ, Nagy ZP, Liu J, Godoy H, Devroey P, Van Steirteghem AC. Conventional in-vitro fertilization versus intracytoplasmic sperm injection for patients requiring microsurgical sperm aspiration. Hum Reprod. 1994;9:1705-9.

13. Palermo G, Joris H, Devroey P, Van Steirteghem AC. Pregnancies after intracytoplasmic injection of single spermatozoon into an oocyte. Lancet. 1992;340:17-8.

14. Tournaye H, Devroey P, Liu J, Nagy Z, Lissens W, Van Steirteghem A. Microsurgical epididymal sperm aspiration and intracytoplasmic sperm injection: a new effective approach to infertility as a result of congenital bilateral absence of the vas deferens. Fertil Steril. 1994;61(6):1045-51.

15. Chen C, Kattera S. Rescue ICSI of oocytes that failed to extrude the second polar body $6 \mathrm{~h}$ post-insemination in conventional IVF. Hum Reprod. 2003;18:2118-21.

16. Xiong S, Han W, Liu JX, Zhang XD, Liu WW, Liu H, et al. Effects of cumulus cells removal after $6 \mathrm{~h}$ co-incubation of gametes on the outcomes of human IVF. J Assist Reprod Genet. 2011;28(12):1205-11.

17. Guo H, Yang J, Zhang C, Li H, Yin B, Gu B, et al. Analysis of clinical data of patients with different outcomes after short-time insemination. Andrologia. 2012;44(1):667-71.

18. Hirsh AV, Mills C, Bekir J, Dean N, Yovich JL, Tan SL. Factors influencing the outcome of in-vitro fertilization with epididymal spermatozoa in irreversible obstructive azoospermia. Hum Reprod. 1994;9(9):1710-6.

19. Bladou F, Grillo JM, Rossi D, Noizet A, Gamerre M, Erny R, et al. Epididymal sperm aspiration in conjunction with in-vitro fertilization 
and embryo transfer in cases of obstructive azoospermia. Hum Reprod. 1991;6(9):1284-7.

20. Yan J, Qinying C, Shuxian L, Fanyu M, Caiping G, Ge S. The impact of semen liquefaction time on the outcomes of in vitro fertilization. J Reprod Contracept. 2012;23(3):169-78.

21. Esteves SC, Miyaoka R, Agarwal A. An update on the clinical assessment of the infertile male. Clinics (Sao Paulo). 2011;66:691-700.

22. Michael Bedford J. Puzzles of mammalian fertilization - and beyond. Int J Dev Biol. 2008;52:415-26.

23. Nagy ZP, Rienzi LF, Ubaldi FM, et al. Effect of reduced oocyte aging on outcome of rescue intracytoplasmic sperm injection. Fertil Steril. 2006;85(4):901-6.

24. Mathieu C, Guérin JF, Cognat M, Lejeune H, Pinatel MC, Lornage J. Motility and fertilizing capacity of epididymal human spermatozoa in normal and pathological cases. Fertil Steril. 1992;57(4):871-6.

25. Van de Velde H, Nagy ZP, Joris H, De Vos A, Van Steirteghem AC. Effects of different hyaluronidase concentrations and mechanical procedures for cumulus cell removal on the outcome of intracytoplasmic sperm injection. Hum Reprod. 1997;12:2246-50.

26. Dandekar P, Aggeler J, Talbot P. Structure, distribution and composition of the extracellular matrix of human oocytes and cumulus masses. Hum Reprod. 1992;7:391-8.

27. Evison M, Pretty C, Taylor E, Franklin C. Human recombinant hyaluronidase (Cumulase) improves intracytoplasmic sperm injection survival and fertilization rates. Reprod Biomed Online. 2009;18(6):811-4.

28. De Vos A, Van Landuyt L, Van Ranst H, et al. Randomized siblingoocyte study using recombinant human hyaluronidase versus bovinederived Sigma hyaluronidase in ICSI patients. Hum Reprod. 2008;23(8):1815-9.

29. Zhu L, Xi Q, Nie R, Chen W, Zhang H, Li Y. Rescue intracytoplasmic sperm injection: a prospective randomized study. J Reprod Med. 2011;56(9-10):410-4.

30. Li M, Haixia J, Yingpu S, et al. Evaluation on clinical outcome of early rescue intracytoplasmic sperm injection after total failure of in vitro fertilization. Prog Obstet Gynecol. 2011;20(3): 218-20.

31. Yamamoto M, Hibi H, Miyake K, Kitagawa T, Asada Y, Suganuma $\mathrm{N}$, et al. Does epididymal length in men with congenital bilateral absence of the vas deferens have a correlation with the fertilization rate of epididymal sperm retrieved by micropuncture technique? Nagoya J Med Sci. 1996;59(1-2):31-5.

32. Patrizio P, Ord T, Silber SJ, Asch RH. Correlation between epididymal length and fertilization rate in men with congenital absence of the vas deferens. Fertil Steril. 1994;61(2):265-8.

33. Suganuma R, Yanagimachi R, Meistrich ML. Decline in fertility of mouse sperm with abnormal chromatin during epididymal passage as revealed by ICSI. Hum Reprod. 2005;20(11):3101-8. 